\title{
Accuracy of Swimming Pool Test Kits
}

\author{
Frankie Tsang ${ }^{1}$, Bobby Sidhu ${ }^{2}$
}

1 Lead Author, B. Tech Student, School of Health Sciences, British Columbia Institute of Technology, 3700 Willingdon Ave, Burnaby, BC V5G 3H2

2 Supervisor, School of Health Sciences, British Columbia Institute of Technology, 3700 Willingdon Ave, Burnaby V5G 3H2

\begin{abstract}
Background: Pool Chemistry is important to allow those using it to feel comfortable through pool water being physically clean and biologically safe. Operators and health inspectors use test kits to ensure that pool water chemistry is correct and will not cause irritation or problems to both the patrons and the pool recirculation system. This study investigates the accuracy of the three commonly used pool test kits available in the market (Taylor, HACH, and ColorQ). Parameters tested are Free Available Chlorine (FAC), Total Chlorine (TC), and $\mathrm{pH}$.

Methods: Using artificial pool water with known concentrations of FAC, TC, and $\mathrm{pH}, 30$ samples were taken for the three different parameter from the three test kits. The indicated concentrations and $\mathrm{pH}$ on the test kits were then recorded and used to compare with the known standards. Results were analyzed using the statistical software NCSS. One sample t-tests were performed to indicate whether or not the test kit as accurate in reading different parameters of pool chemistry.
\end{abstract}

Results:

Taylor Test Kit: Readings for FAC (2.6ppm) showed 2.4ppm, TC (2.7ppm) showed 2.43ppm, and pH (7.1) showed 7.1.

HACH Test Kit: Readings for FAC (2.8ppm) showed 3.5ppm, TC (3.0ppm) showed 3.5ppm, and pH (7.1) showed 6.97.

ColorQ Test Kit: Readings for FAC (2.7ppm) showed 3.0ppm, TC (3.0ppm) showed 3.0ppm, and pH (7.0) showed 6.96.

Conclusion: All three test kits have accurate readings for $\mathrm{pH}$ levels. However, the test kits do not provide accurate readings for FAC and TC which would make it difficult to calculate CC in pool waters. Although the FAC and TC readings are inaccurate, they are able to provide operators and health inspectors with brief information regarding pool water chemistry.

Keywords: Pool Test Kit, Swimming Pools, Chlorine, pH, Taylor, ColorQ, HACH

\section{Introduction}

Swimming is an activity that can be done leisurely or competitively and is suitable for all ages. Not only is swimming fun, it's also beneficial in terms of physical and mental health (Centers for Disease Control and Prevention, 2013a). Pool chemistry plays an important role in ensuring that pool water is biologically safe and physically clean for those who use it. Swimming pools may pose a risk when the water chemistry within pools do not meet legislative standards. This may cause microbes to be able to thrive causing serious illnesses or irritation to swimmers. Regulations are developed to ensure operators will maintain the pools so that it will be safe and clean for people to use. The BC Pool Regulation states that the chemistry of the pool water must be tested daily by operators (Pool Regulation, 2012) with factors to be tested including $\mathrm{pH}$ level, alkalinity, disinfectants, and combined chlorine. In addition to regulations, health inspectors play an important role in working with pool operators to ensure that the facilities comply with the regulations and is safe for public use. In order to test pool water chemistry, pool test kits are available for both operators and inspectors to use. However, it has been noted that there may be discrepancies between the readings of different test kits with respect to assessing water chemistry. Due to the discrepancies, it may be hard to determine the course of action needed to be taken when pool water is inspected. It is important to ensure that the pool test kits are giving the user a correct reading of pool water parameters. 


\section{Literature Review}

\section{Injuries and Illnesses}

Although swimming is an activity that everyone can enjoy, it can also be dangerous to those who swim in pools that do not regulations standards. Injuries or illnesses can arise if pools are not treated so that they are clean and safe from physical and biological hazards. Physical injuries such as entrapment and drowning can prove fatal if left unnoticed (Centers for Disease Control, 2014a). In addition, Contamination of pool water via fecal accidents may also happen in water. According to the Centers for Disease Control and Prevention (2013b), recreational water illnesses can be transferred through contacting or ingesting contaminated water and breathing in mists which contain pathogens.

\section{Microbes Present in Pool Water}

There are many opportunities for microbes to enter and contaminate pool water. Outdoor pools are constantly at risk of contamination as they are constantly being exposed to the environment around them. Animals, plants, and also the air may contaminate the pool water. Although indoor pools do not have the same factors that contribute to the contamination of outdoor pools, indoor pools must also be carefully monitored to ensure that patrons are not exposed to pathogens. According to the Centers for Disease Control (2014b) and Prevention and the Pool \& Spa Operator ${ }^{\mathrm{TM}}$ Handbook (2014a), some of the top causes for water outbreaks include: Cryptosporidium, Pseudomonas, Legionella, Giardia, Shigella, E. coli and Norovirus. Many of the above causes for water outbreaks can be prevented through the proper use of disinfectants in pool waters. A common way for microorganisms to contaminate water would be through fecal contamination (World Health Organization, 2006).

In 2001, 11 people were reported to have diarrhea in Iowa. It was identified that Shigella was present in the stool samples of those infected. Investigation showed that all patients had been exposed to a fill and drain wading pool which had no recirculation nor disinfection system. Although water in the pool was emptied daily, the pool basin was only cleaned twice a week. The lack of cleaning of the pool was concluded to be the reason for the outbreak (Centers for Disease Control and Prevention, 2001).

A number of children have been tested positive for E. coli O157 in 2004, after swimming in a local pool. It was later identified that the pool disinfection system was not working causing a rise in bacterial numbers while no chlorine was pumped into the pool water to disinfect the contamination (Verma et al., 2007).

In order to remove and kill pathogens in water, disinfectant must be used to keep pool water physically clean and biologically safe. Pool chemistry plays an important part of disinfecting pools to ensure that patrons have a safe environment to enjoy swimming in. In British Columbia, the Pools Regulation indicates the minimum concentrations of disinfectants (chlorine or bromine) that pools are required to have in order to kill the microbes that are in the pool water.

\section{BC Regulations}

The British Columbia Pool Regulation states requirements and standards for pools to comply with. Free available chlorine (FAC), total chlorine (TC), alkalinity, and $\mathrm{pH}$ levels all have standards for pool operators to follow. The regulation states that the $\mathrm{pH}$ levels must be within 7.2 to 7.8 , alkalinity within 80 to 120 parts per million (ppm), combined chlorine must be less than $1 \mathrm{ppm}$. The minimum concentrations of disinfectants are also given within schedule 3 of the regulations. Pools must follow the standards set by the regulation depending on the type of disinfectant the pools run on. Free available chlorine must be at least $0.5 \mathrm{ppm}$, chlorine cyanurate at $1.0 \mathrm{ppm}$, and Bromine at $1.5 \mathrm{ppm}$ if the pool water is less than $30^{\circ} \mathrm{C}$. If the pool water is above $30^{\circ} \mathrm{C}$, free available chlorine must be at least $1.5 \mathrm{ppm}$, chlorine cyanurate at $2.0 \mathrm{ppm}$, and Bromine at $2.5 \mathrm{ppm}$. Pool operators must also test the pools twice a day with regards to $\mathrm{pH}$ levels, combined chlorine concentration and disinfectant concentrations while alkalinity of pool water is to be tested weekly (Pool Regulation, 2012).

The BC Public Health Act delegates health inspectors the powers to inspect pools in accordance to the BC Pools Regulation (BC Public Health Act, 2014). The duties of a health inspector includes inspection and approval of 
swimming pool applications and construction. Health inspectors are provided with pool test kits to allow them to correctly measure whether the pool chemistry meets the requirements of the regulations. If the reading of the pool chemistry does not meet the requirements, the inspectors will have to take the necessary actions to ensure that the public is protected from possible injuries that may result from swimming in the pool.

\section{Chlorine}

Chlorine and bromine are currently the two types of primary disinfectant that used to disinfect pool water. Among the two, chlorine is the most common disinfectant used to treat pool water (Pool \& Spa Operator ${ }^{\mathrm{TM}}$ Handbook, 2014b) while bromine may be used in smaller pool systems or spas. Reasons why a majority of the pools use chlorine as a disinfectant may be due to its stability in water (Sidhu, 2014a) and its effectiveness against certain pathogens such as E. coli in water (Koski, Stuart, \& Ortenzio, 1966). Chlorine in water consist of three different types, total chlorine (TC), free available chlorine (FAC), and combined chlorine (CC). Among the three types, combined chlorine cannot be measured through the use of a DPD pool test kit and must be calculated through the following equation: Free Chlorine + Combined Chlorine $=$ Total Chlorine (Sidhu, 2014a) Health inspectors will have to determine the concentration of both the TC and FAC in order to determine the concentration of CC. The Pool Regulation states that combined chlorine must be tested twice daily and maintained at a concentration less than 1 ppm (Pool Regulation, 2012). High combined chlorine concentrations in water may cause irritation to those swimming. The present of combined chlorines may also cause the pool water to turn milky which may cause a physical hazard as lifeguards are unable to see the bottom of the pool clearly to identify problems within the water (Sidhu, 2014a). Ways to remove combined chlorine include adding free chlorine into the pools though the process of superchlorination (Pool \& Spa Operator ${ }^{\mathrm{TM}}$ Handbook, 2014c). Swimming can then be resumed when the chlorine concentration remains below the regulation standards (World Health Organization, 2006).
pH

The $\mathrm{pH}$ scale measures if an aqueous solution is acidic or basic. The scale runs from 0 to 14 where 0 is the acidic, 14 is basic, and 7 is neutral. Water has a natural $\mathrm{pH}$ of 7 which would indicate that pool water should also be kept at a $\mathrm{pH}$ of 7 . However, regulations state that $\mathrm{pH}$ should be kept between a $\mathrm{pH}$ of 7.2 to 7.8 (Pool Regulation, 2012). Reason for the regulation to keep $\mathrm{pH}$ slightly basic is due to the $\mathrm{pH}$ of the human eye. The human eye has a $\mathrm{pH}$ level of 7.5 and pool water is kept at $\mathrm{pH}$ of 7 , irritation may start to occur as there is a significant difference between the two $\mathrm{pHs}$ (Sidhu, 2014b). If pool water is too acidic, a more basic material would be added to increase the $\mathrm{pH}$ level of water and vice versa. However, disinfectants may have an influence on $\mathrm{pH}$ as well due to the nature of the chemicals that are used. For example, Sodium hypochlorite is basic and would raise the $\mathrm{pH}$ of pool water while Trichlor is more acidic leading to the lowering of $\mathrm{pH}$ if added (Pool \& Spa Operator ${ }^{\mathrm{TM}}$ Handbook, 2014b).

\section{Pool Test Kits}

There are currently numerous pool test kits on the market that test for basic pool chemistry. The test kits currently being used by pool operators and health authorities include the Taylor (Taylor Company, 2014a), HACH (HACH Company, 1991), and Colour Q (LaMotte Company, 2014a) test kit. Although the test kits are used throughout the industry, it is uncertain whether all the test kits would yield the same results when being tested on the same body of pool water. Research on the accuracy of pool test kits has been done before by a graduate that attended the British Columbia Institute of Technology Environmental Health Program in 2004 (Johal). However, due to the study being dated over a decade ago, new test kits have entered the market and begun to replace the old test kits used back in 2004. For example, the Hack Pool Master test kit was once used by the health authority, but due to the emerging of the Colour Q test kits, the Hack Pool Master kit is being replaced. Digital readings on the new Colour Q are a quicker alternative to reading of pool chemistry compared to comparator disk where the user has to determine the results with the naked eye. 
Graduate students from the environmental health program in BCIT have found that there are discrepancies between different test kits on the same body of water during their inspections. Different readings were found during an inspection where discrepancies were found in the readings for FAC and TC. The different readings on the pool test kits would cause calculation for CC to differ and thus, the corrective actions could not be taken as the concentrations for CC could not be determined through the varied results.

\section{Public Health Significance}

Misinterpreted results from the pool test kits during inspection can lead to numerous problems and may pose health problems to the public if not treated in a proper way. As mentioned earlier, operators and the health authority may not be able to determine what corrective actions should done to return the pool to regulation standards. The different indicated results would also hinder the decision made by the health inspector or the pool management team on whether the pool should be closed down or not. Problems such as irritation or ingestion of contaminated water on patrons/public may also arise as a result from not closing pools that do not meet regulation requirement. Incorrect readings of pool water containing chlorine concentrations lower than regulation standards may mislead operators and inspectors into thinking that the pool is safe while pathogens and microbes are not being killed sufficiently. Incorrect readings of chlorine concentrations may also lead to calculating the wrong combined chlorine levels which may lead to physical hazards present in the pool turning the water milky due to high concentrations of combined chlorine. This may lead to serious injuries or delayed first aid treatment due to lifeguards unable to identify the activities within the pool. It is important to ensure that pool test kits provide the correct data to allow operators and inspectors to do what is correct under the specific circumstances.

\section{Purpose}

The purpose of this research is to identify the accuracy of the different pool test kits with respect to alkalinity, free chlorine, total chlorine, and $\mathrm{pH}$ levels of a known value. The research will determine if all pool test kits are the same and interchangeable for pool operators and inspectors to use.

\section{Materials Used}

In order to obtain results of the accuracy of pool test kits, tests were conducted with the chosen pool test kits. The pool test kits tested were the Taylor test kit, HACH test kit, and the ColorQ test kit. The three different test kits are commonly used by both the health authorities and pool operators to ensure that the pool quality is within regulation standards. The pool test kit were tested on accuracy on the main parameters that health authorities test for during a regular inspection which are: Free Available Chlorine (FAC), Total Chlorine (TC), and pH (Pool Regulation, 2012).

In addition to the three test kits that were used, the HACH Pocket Colorimeter II was also used to provide an actual reading of FAC, $\mathrm{TC}$, and $\mathrm{pH}$ of the artificial pool water.

Calibration tools for the colorimeter included a secondary chlorine standards kit produced by $\mathrm{HACH}$ and a $7.0 \mathrm{pH}$ buffer solution to ensure that the colorimeter provides accurate information.

The tests was done with water left at room temperature below 30 degrees Celsius. As a result, the regulation standard for the minimum amount of FAC would be 0.5 ppm of chlorine (Pool Regulation, 2012).

The test kits were obtained from the Environmental Health lab in BCIT. Please refer to Table 1 for the list of materials used in this study. 
Table 1: Materials Used for the Study

\section{Materials}

\begin{tabular}{|c|c|c|}
\hline \multirow[t]{5}{*}{ Taylor Test Kit } & \multicolumn{2}{|c|}{ Comparator Block (w/ cap) } \\
\hline & \multirow[t]{4}{*}{ Solutions } & $\mathrm{R}-0001$ \\
\hline & & R-0002 \\
\hline & & R-0003 \\
\hline & & R-0004 \\
\hline \multirow[t]{7}{*}{ HACH Test Kit } & \multicolumn{2}{|c|}{ Viewing Tube (w/ cap) } \\
\hline & \multicolumn{2}{|c|}{ Colour Comparator } \\
\hline & \multicolumn{2}{|c|}{ Comparator Disk (Chlorine) } \\
\hline & \multicolumn{2}{|c|}{ Comparator Disk (pH) } \\
\hline & \multicolumn{2}{|c|}{ 5mL DPD Free Chlorine Reagent } \\
\hline & \multicolumn{2}{|c|}{ 5mL DPD Total Chlorine Reagent } \\
\hline & \multicolumn{2}{|c|}{ Phenol Red Indicator } \\
\hline \multirow[t]{7}{*}{ ColorQ Test Kit } & \multirow[t]{4}{*}{ Solutions } & DPD 1A \\
\hline & & DPD 1B \\
\hline & & DPD 3 \\
\hline & & $\mathrm{pH}$ \\
\hline & \multicolumn{2}{|c|}{ Test Tube (w/ cap) } \\
\hline & \multicolumn{2}{|c|}{ ColorQ Meter } \\
\hline & \multicolumn{2}{|c|}{ Sample Bottle } \\
\hline \multirow{3}{*}{$\begin{array}{l}\text { HACH Pocket } \\
\text { Colorimeter }\end{array}$} & \multicolumn{2}{|c|}{ DPD Secondary Standards Kit } \\
\hline & \multicolumn{2}{|c|}{ 7.0 pH Buffer Solution } \\
\hline & \multicolumn{2}{|c|}{ Sample Cell (w/ cap) } \\
\hline \multicolumn{3}{|l|}{ 1mL Pipets } \\
\hline \multicolumn{3}{|c|}{ 200mL \& 2000mL Beakers } \\
\hline \multicolumn{3}{|c|}{ Clorox $^{\circledR}$ Disinfecting Bleach } \\
\hline \multicolumn{3}{|l|}{ Kimwipes } \\
\hline \multicolumn{3}{|l|}{ Potable Water } \\
\hline \multicolumn{3}{|l|}{ Notebook } \\
\hline
\end{tabular}

In order to test for the accuracy of pool test kits, the artificial pool water was made within the BCIT labs (SW1 1230) following parameters based on the BC pool Regulation: FAC concentration would be at $3.0 \pm 0.5 \mathrm{ppm}$, TC concentration would be at $3.0 \pm 0.5 \mathrm{ppm}$, and $\mathrm{pH}$ level would be at $7.2 \pm 0.2$.

\section{Standard Methods}

The desired chlorine levels in the artificial water was obtained through mixing $5 \mathrm{~mL}$ of chlorine with $95 \mathrm{~mL}$ of potable water in a $200 \mathrm{~mL}$ beaker. $1 \mathrm{~mL}$ of solution was then transferred into a $2000 \mathrm{~mL}$ beaker mixed with $1999 \mathrm{~mL}$ of potable water. The result would be artificial pool water that would have a chlorine concentration at $3.0 \pm 0.5 \mathrm{ppm}$

To ensure that the pool water had the correct parameter concentration, the HACH Pocket
Colorimeter II was used to identify the correct concentration of FAC, TC, and $\mathrm{pH}$.

To calibrate for chlorine, the four different vials in the secondary standards kit will be used. First, the vial labelled 'BLANK' will be used to zero the colorimeter. The vial labelled 'STD 1' will then be measured, which should yield results at $2.2 \pm 0.2 \mathrm{ppm}$, 'STD 2' should yield results at $3.7 \pm 0.3 \mathrm{ppm}$, and 'STD 3' should yield results at $6.6 \pm 0.6 \mathrm{ppm}$ (HACH Company, 2013). For the calibration of $\mathrm{pH}, 5 \mathrm{~mL}$ of water will be obtained and placed into the sample cell. $0.5 \mathrm{~mL}$ of $7.0 \mathrm{pH}$ buffer solution will then be pipetted into the cell and stirred. $0.5 \mathrm{~mL}$ of Phenol Red Solution will also be pipetted into the cell. The cell will then be placed into the colorimeter for reading and should yield results at the range of $7.0 \pm 0.2$ (HACH Company, 2013). If any of the calibration tests fail, the colorimeter should not be used and should be returned to the manufacturer for checkup.

To test for FAC levels for the Taylor Kit, the small comparator tube will be filled to the $9 \mathrm{~mL}$ water mark. 5 drops of 'R-0001' and 5 drops of 'R-0002' will then be added into the comparator tube and mixed. The colour of the solution will then be matched with the colour on the comparator tube to determine the FAC levels. For TC levels, 5 drops of 'R-0003' will be added to the previous solution and the colour of the solution will be matched with the comparator tube to find the TC levels. As for $\mathrm{pH}$, water will be added to the large comparator tube to the 44 mL mark and 5 drops of 'R-0004' will be added. The colour of the solution will then be matched with the colour on the comparator tube to determine the $\mathrm{pH}$ of the water (Taylor Company, 2014b).

To test for FAC levels for the ColorQ Kit, fill the provided test tube with sample water to the $5 \mathrm{~mL}$ line and place it into the ColorQ meter. Press the button on the meter. When 'bLA' appears, press the button again to Zero/Blank the meter. Add 5 drops of 'DPD 1A' and 5 drops of 'DPD 1B' to the test tube. Insert the tube into the meter and press the button to read the 'FCL' for free chlorine. For total chlorine, remove the same tube and add in 5 drops of 'DPD 3' into the tube. Insert the tube into the meter and press the button to read the ' $\mathrm{tCL}$ ' for total chlorine. For $\mathrm{pH}$, fill a clean tube to the $5 \mathrm{~mL}$ line and add 5 drops of ' $\mathrm{pH}$ ' solution. Insert the tube into the 
meter and press the button to read ' $\mathrm{PH}$ ' for $\mathrm{pH}$ levels (LaMotte Company, 2014b).

To test for FAC for the HACH Kit, fill a viewing tube to the $5 \mathrm{~mL}$ mark and place it on the left opening of the comparator. Then fill another tube to the same $5 \mathrm{~mL}$ mark and add in one DPD Free Chlorine Reagent Powder pillow $(5 \mathrm{~mL})$. Place the sample tube on the top right opening of the comparator. Hold the comparator up to a light source and view through the openings in front and rotate the comparator disc (chlorine) until a colour match is obtained. In order to test for TC, the procedures are the same as FAC except the DPD Total Chlorine Reagent Powder pillow is used instead. To test for $\mathrm{pH}$, two viewing tubes will be filled to the $5 \mathrm{~mL}$ mark and four drops of Phenol Red Indicator Solution will be added into one of the tubes. The viewing tube with the clear solution will then be placed in the left opening while the other viewing tube will be placed on the left opening of the comparator. Hold the comparator up to a light source and view through the openings in front and rotate the comparator disc $(\mathrm{pH})$ until a colour match is obtained (HACH Company, 1991).

30 samples were taken for the each of the three parameters for each test kit. For example, 30 water samples were taken and tested on FAC for the Taylor test kit and 30 samples would to be tested for TC for the Taylor test kit. FAC and TC were recorded in ppm while $\mathrm{pH}$ had no units. After testing for each test kit, the pool water was tested again with the calibrated pocket colorimeter to ensure that the parameter concentrations remained the same. If there was a difference in the water sample at the beginning, the sample was adjusted so that it would meet the sample criteria listed above.

The different ranges indicated in the water sample for TC $(3.0 \pm 0.3 \mathrm{ppm})$ and FAC $(3.0 \pm$ $0.5 \mathrm{ppm}$ ) was because water was created at the beginning of each testing session. The exact amount of chlorine concentration was difficult to adjust. In addition, after time, FAC would decrease as it is being used up.

After all the data has been collected, it was analyzed with the statistical software NCSS.

\section{Reliability and Validity \\ Reliability and validity plays an \\ important part of every experiment. Being able}

to yield the same results for each test kit ensures that when inspectors or pool operators use the pool test kit to test for pool chemistry, the test kit would always indicate the same results no matter how many times the test would be done (Heacock \& Sidhu, 2014a). The solution provided for each test kit was obtained from the manufacturer which indicated that each test should yield the same results no matter how many times it will be done. To ensure the reliability of the instruments, each parameters were tested 30 times with the same test kit.

The main purpose of this study was to ensure that the results the test kit yields were valid. This means that the test kit should provide a valid measure of the parameters that are being tested on (Heacock \& Sidhu, 2014a). In order to know whether or not the data is valid or not, the samples of water were calibrated first with a calibrated instrument to allow us to compare the validity of the results from the calibrated instrument and the three different pool test kits.

\section{Inclusion and Exclusion Criteria}

The pool test kits being used in this study cannot be expired. The majority of the test kits have a shelf life of 1 year therefore all the test kits used in this study were manufactured within one year before the data collection (LaMotte Company, 2014d, Taylor Company, 2014c). Water used within this study was municipal water obtained in Metro Vancouver. The water used ensured that the test would apply to all the pools within Metro Vancouver. Distilled water should not be used to produce the sample pool water. The pool water within public and private pools within Metro Vancouver use municipal water which may contain different minerals. The use of distilled water may cause the readings of the test kits to be inaccurate.

\section{Statistical Analysis}

Data obtained from measuring the three parameters are all numerical data. Since the measurement of the data was on a continuum as ppm and $\mathrm{pH}$, the numeric data was continuous as opposed to discrete. A one sample t-test was performed to compare the test results to a known standard. The software NCSS would be used to calculate the statistics of the tests. 


\section{Collected Data}

Tables 2-4 indicate the results that were obtained from the study for each test kit. The software NCSS was then used to analyze the given results to determine the accuracy of each test kit on the tested parameters.

Table 2: FAC, TC \& pH readings for Taylor Kit

\begin{tabular}{|c|c|c|c|}
\hline \multicolumn{4}{|c|}{ Taylor Test Kit } \\
\hline & $\begin{array}{c}\mathrm{FAC}=2.6 \\
(\mathrm{ppm})\end{array}$ & $\begin{array}{c}\mathrm{TC}=2.7 \\
(\mathrm{ppm})\end{array}$ & $\mathrm{pH}=7.1$ \\
\hline 1 & 2.5 & 2.5 & 7.1 \\
\hline 2 & 2.5 & 2.5 & 7.2 \\
\hline 3 & 2.5 & 2.5 & 7.2 \\
\hline 4 & 2.5 & 2.5 & 7.2 \\
\hline 5 & 2.0 & 2.0 & 7.1 \\
\hline 6 & 2.5 & 2.0 & 7.1 \\
\hline 7 & 2.5 & 2.5 & 7.1 \\
\hline 8 & 2.5 & 2.5 & 7.1 \\
\hline 9 & 2.5 & 2.5 & 7.1 \\
\hline 10 & 2.5 & 2.5 & 7.0 \\
\hline 11 & 2.5 & 2.5 & 7.0 \\
\hline 12 & 2.5 & 2.0 & 7.1 \\
\hline 13 & 2.5 & 2.5 & 7.1 \\
\hline 14 & 2.5 & 2.5 & 7.1 \\
\hline 15 & 2.5 & 2.5 & 7.1 \\
\hline 16 & 2.0 & 3.0 & 7.1 \\
\hline 17 & 2.5 & 3.0 & 7.1 \\
\hline 18 & 2.5 & 2.5 & 7.1 \\
\hline 19 & 2.0 & 2.0 & 7.1 \\
\hline 20 & 2.5 & 2.5 & 7.1 \\
\hline 21 & 2.0 & 2.5 & 7.1 \\
\hline 22 & 2.5 & 2.5 & 7.1 \\
\hline 23 & 2.5 & 2.0 & 7.1 \\
\hline 24 & 2.0 & 2.0 & 7.1 \\
\hline 25 & 2.0 & 2.5 & 7.1 \\
\hline 26 & 2.5 & 2.5 & 7.1 \\
\hline 27 & 2.5 & 2.5 & 7.1 \\
\hline 28 & 2.5 & 2.5 & 7.1 \\
\hline 29 & 2.5 & 2.5 & 7.1 \\
\hline 30 & 2.5 & 2.5 & 7.1 \\
\hline Mean & 2.4 & 2.43 & 7.1 \\
\hline $\begin{array}{c}\text { Standard } \\
\text { Deviation }\end{array}$ & 0.203 & 0.254 & 0.045 \\
\hline
\end{tabular}

\section{Taylor Test Kit}

The actual water to be tested showed 2.6ppm on the colorimeter for FAC and mean of the results of the test kit was 2.4ppm and median was 2.5ppm. Standard deviation of FAC is 0.203 and results ranged from 2.0ppm to 2.5ppm. For TC, the colorimeter showed 2.7ppm while the mean from the test kit was calculated to be 2.433ppm and median to be 2.5 . Standard deviation was 0.254 and results ranged from 2.0 to $3.0 \mathrm{ppm}$. Finally, $\mathrm{pH}$ was indicated at 7.1 on the pocket colorimeter while the test kit had a mean of 7.12 and median of 7.1. Standard deviation of $\mathrm{pH}$ is 0.045 and results ranged from 7.0 to 7.2 .
Table 3: FAC, TC \& pH readings for HACH Kit

\begin{tabular}{|c|c|c|c|}
\hline \multicolumn{4}{|c|}{ HACH Test Kit } \\
\hline & $\begin{array}{c}\mathrm{FAC}=2.8 \\
(\mathrm{ppm})\end{array}$ & $\begin{array}{c}\mathrm{TC}=3.0 \\
(\mathrm{ppm})\end{array}$ & $\mathrm{pH}=7.1$ \\
\hline 1 & $3.5^{+}$ & $3.5^{+}$ & 6.9 \\
\hline 2 & $3.5^{+}$ & $3.5^{+}$ & 6.9 \\
\hline 3 & $3.5^{+}$ & $3.5^{+}$ & 7.0 \\
\hline 4 & $3.5^{+}$ & $3.5^{+}$ & 7.0 \\
\hline 5 & $3.5^{+}$ & $3.5^{+}$ & 7.0 \\
\hline 6 & $3.5^{+}$ & $3.5^{+}$ & 7.0 \\
\hline 7 & $3.5+$ & $3.5^{+}$ & 6.9 \\
\hline 8 & $3.5^{+}$ & $3.5^{+}$ & 6.9 \\
\hline 9 & $3.5^{+}$ & $3.5^{+}$ & 7.0 \\
\hline 10 & $3.5^{+}$ & $3.5^{+}$ & 7.0 \\
\hline 11 & $3.5^{+}$ & $3.5^{+}$ & 7.0 \\
\hline 12 & $3.5^{+}$ & $3.5^{+}$ & 7.0 \\
\hline 13 & $3.5+$ & $3.5+$ & 7.0 \\
\hline 14 & $3.5^{+}$ & $3.5^{+}$ & 7.0 \\
\hline 15 & $3.5^{+}$ & $3.5+$ & 6.9 \\
\hline 16 & $3.5^{+}$ & $3.5+$ & 6.9 \\
\hline 17 & $3.5^{+}$ & $3.5^{+}$ & 7.0 \\
\hline 18 & $3.5^{+}$ & $3.5^{+}$ & 7.0 \\
\hline 19 & $3.5^{+}$ & $3.5+$ & 6.9 \\
\hline 20 & $3.5^{+}$ & $3.5^{+}$ & 6.9 \\
\hline 21 & $3.5+$ & $3.5^{+}$ & 7.0 \\
\hline 22 & $3.5^{+}$ & $3.5+$ & 7.0 \\
\hline 23 & $3.5^{+}$ & $3.5^{+}$ & 7.0 \\
\hline 24 & $3.5^{+}$ & $3.5^{+}$ & 7.0 \\
\hline 25 & $3.5^{+}$ & $3.5+$ & 7.0 \\
\hline 26 & $3.5^{+}$ & $3.5^{+}$ & 7.0 \\
\hline 27 & $3.5^{+}$ & $3.5^{+}$ & 7.0 \\
\hline 28 & $3.5+$ & $3.5+$ & 7.0 \\
\hline 29 & $3.5+$ & $3.5^{+}$ & 7.0 \\
\hline 30 & $3.5^{+}$ & $3.5^{+}$ & 7.0 \\
\hline Mean & 3.5 & 3.5 & 6.97 \\
\hline $\begin{array}{c}\text { Standard } \\
\text { Deviation }\end{array}$ & 0 & 0 & 0.045 \\
\hline
\end{tabular}

Note: The comparator disc was only able to read up to 3.5ppm therefore 3.5+ indicates that the results are greater than $3.5 \mathrm{ppm}$.

\section{HACH Test Kit}

The actual water to be tested showed 2.8ppm on the colorimeter for FAC and mean of the results of the test kit was 3.5ppm and median was 3.5ppm. Standard deviation of FAC is 0 . For TC, the colorimeter showed 3.0ppm while the mean from the test kit was calculated to be 3.5ppm and median to be 3.5. Standard deviation was 0 . Finally, $\mathrm{pH}$ was indicated at 7.1 on the pocket colorimeter while the test kit had a mean of 6.97 and median of 7. Standard deviation of $\mathrm{pH}$ is 0.045 and results ranged from 6.9 to 7.0 . 
Table 4: FAC, TC \& pH readings for ColorQ Kit

\begin{tabular}{|c|c|c|c|}
\hline \multicolumn{4}{|c|}{ HACH Test Kit } \\
\hline & $\begin{array}{c}\mathrm{FAC}=2.8 \\
(\mathrm{ppm})\end{array}$ & $\begin{array}{c}\mathrm{TC}=3.0 \\
(\mathrm{ppm})\end{array}$ & $\mathrm{pH}=7.1$ \\
\hline 1 & 2.98 & 2.98 & 6.9 \\
\hline 2 & 2.99 & 3.01 & 7.0 \\
\hline 3 & 3.05 & 3.07 & 7.0 \\
\hline 4 & 3.05 & 3.05 & 7.0 \\
\hline 5 & 3.08 & 3.10 & 6.9 \\
\hline 6 & 3.07 & 3.07 & 7.0 \\
\hline 7 & 2.89 & 2.96 & 7.0 \\
\hline 8 & 3.01 & 3.01 & 6.9 \\
\hline 9 & 3.04 & 3.04 & 6.9 \\
\hline 10 & 3.00 & 3.06 & 7.0 \\
\hline 11 & 3.02 & 3.02 & 6.9 \\
\hline 12 & 2.96 & 2.96 & 7.0 \\
\hline 13 & 3.06 & 3.06 & 6.9 \\
\hline 14 & 2.95 & 2.99 & 7.0 \\
\hline 15 & 3.02 & 3.02 & 7.0 \\
\hline 16 & 2.97 & 2.97 & 6.9 \\
\hline 17 & 3.02 & 3.02 & 7.0 \\
\hline 18 & 2.98 & 2.98 & 7.0 \\
\hline 19 & 3.01 & 3.01 & 6.9 \\
\hline 20 & 2.99 & 2.99 & 7.0 \\
\hline 21 & 2.96 & 2.96 & 7.0 \\
\hline 22 & 2.96 & 2.97 & 7.0 \\
\hline 23 & 2.92 & 2.93 & 6.9 \\
\hline 24 & 2.98 & 2.98 & 7.0 \\
\hline 25 & 2.99 & 2.99 & 6.9 \\
\hline 26 & 2.98 & 3.00 & 7.0 \\
\hline 27 & 3.03 & 3.03 & 7.0 \\
\hline 28 & 2.96 & 2.96 & 7.0 \\
\hline 29 & 2.90 & 2.90 & 6.9 \\
\hline 30 & 2.94 & 2.94 & 7.0 \\
\hline Mean & 2.99 & 3.00 & 6.96 \\
\hline $\begin{array}{l}\text { Standard } \\
\text { Deviation }\end{array}$ & 0.048 & 0.046 & 0.049 \\
\hline
\end{tabular}

\section{ColorQ Test Kit}

The actual water to be tested showed 2.7ppm on the colorimeter for FACand the mean of the results of the test kit was 2.99ppm and median was 2.99ppm. Standard deviation of FAC is 0.048 and results ranged from 2.89ppm to 3.08ppm. For TC, the colorimeter showed $3.0 \mathrm{ppm}$ while the mean from the test kit was calculated to be $3.00 \mathrm{ppm}$ and median to be 2.995. Standard deviation was 0.046 and results ranged from 2.93 to $3.10 \mathrm{ppm}$. Finally, $\mathrm{pH}$ was indicated at 7.0 on the pocket colorimeter while the test kit had a mean of 6.96 and median of 7 . Standard deviation of $\mathrm{pH}$ is 0.049 and results ranged from 6.9 to 7.0 .

\section{Discussion}

This study was done to determine whether or not pool test kits used in the market would yield accurate results for the different pool parameters that are being tested on. Results from this study shows that the all the three test kits that were tested were not accurate in some of the parameters that they were tested on. After analyzing the descriptive statistics results, we are able to determine that:

1. The Taylor Test Kit only showed accurate results on $\mathrm{pH}$,

2. The HACH Test Kit only showed accurate results for $\mathrm{pH}$, and

3. The ColorQ Test Kit showed accurate results for $\mathrm{TC}$ and $\mathrm{pH}$.

Through the findings, we are able to see that problems may arise now with the three test kits unable to yield inaccurate results of pool chemistry. It would be difficult for pool operators and health inspectors to determine if the pool is physically clean and biologically safe for patrons to swim in. The readings for FAC for all test kits showed results that were different from the actual FAC of the artificial pool water. Without the accurate readings for FAC, combined chlorine levels cannot be calculated correctly. As a result, operators and inspectors will not be able to conduct the proper corrective actions to the pool water.

On the other hand, we are able to determine that the $\mathrm{pH}$ tests for all the test kits are fairly accurate as the results are all within a range of \pm 0.1 . This would ensure that the $\mathrm{pH}$ of pool waters will be correctly monitored to provide a safe environment for swimmers.

With regards to the Taylor and ColorQ Test Kit, although the statistical data indicated that there is a different with the chlorine readings and the water chlorine levels, it is still able to give operators and inspectors a good overview of how good the pool chemistry is. The data collected from the two test kits with regards to chlorine are within the range of $\pm 0.5 \mathrm{ppm}$. Although the range may make it hard to calculate the combined chlorine levels, the readings may give information to operators and inspectors the quality of the pool water.

\section{Limitations}

There are many different aspects of this study that can be improved to obtain more accurate results with regards to the accuracy of pool test kits. These includes looking at alternate methods such as the use of pool water from swimming pools instead of artificial pool water that was synthesized within the lab room. The artificial pool water created in the lab does 
not have all the physical and chemical properties of actual swimming pool water. In addition to chlorine and $\mathrm{pH}$, swimming pools also have parameters such as calcium hardness and alkalinity. The artificial water does not have these factors. The use of actual swimming pool water may increase the accuracy of the results due to the effects of the different chemicals' interactions. For example, the change in $\mathrm{pH}$ levels may cause a change in the indicator solution with chlorine and provide a different (maybe more accurate) reading.

The three test kits that were used in this study may also have its own limitation that may cause results to contain errors and bias to occur. Color Q

The overall use of the ColorQ Test Kit was simple when compared to the other two test kits. There are similarities between the Taylor and ColorQ in terms of the different vials of solution. One of the main features of the ColorQ is the photometer within the test kit. The photometer helps indicate the concentration of the parameters without the use of the human eye which eliminates the subjectivity of the Taylor and HACH Test Kit. Calibration of the instrument is conducted every time it is turned on as the user is required to ZERO the instrument with a test tube of pool water. With results that was obtained from this study, we have found that the chlorine concentrations were consistently incorrect. The incorrect results may be due to the lens of the photometer being contaminated and thus, hindering the results. However, the lens of the ColorQ photometer could not be cleaned as the instrument did not provide any cleaning protocols.

Taylor

The Taylor Test Kit uses a comparator block to determine the concentration of Chlorine and $\mathrm{pH}$ levels. The comparator block requires the use of the human eye to determine the chlorine, bromine and $\mathrm{pH}$ levels of the pool water. For chlorine levels, the comparator block consists of concentrations $0.5,1,1.5,2,3$, and 5 each with its own shade of colour to indicate the level of chlorine. Limitation of this test kit may occur when the colour of the tested water lies between two shades. For example, during the test for FAC, the water concentration of FAC was 2.6. The colour indicated on the comparator tube was between 2 and 3 . With the use of the human eye, the colour sometimes leaned towards 2ppm but if the angle of the comparator block changed, it felt like it showed 3ppm. The subjectivity present with this test kit made it difficult to determine the accurate results. In addition, if the colour were to be between two shades, it is impossible to determine the accurate concentration or $\mathrm{pH}$ level of the water. The difference between 2ppm and 3ppm may be the determining factor in whether or not the pool should be closed or not.

$\mathrm{HACH}$

The HACH test kit uses a comparator disk to determine the different levels of chlorine and $\mathrm{pH}$. Similarly to the Taylor Kit, the use of such disk is very subjective as it requires the human eye to make the judgment on what the concentration of chlorine or $\mathrm{pH}$ should be. However, there is a difference between the two kits. The HACH test kit is easier at identifying the correct concentration as the disk provides a band with gradual levels of a certain colour. Unlike the Taylor Test Kit, the band allows a more accurate reading towards the chlorine and $\mathrm{pH}$ levels. However, the highest concentration for Chlorine only reaches 3.5ppm. For pools with chlorine levels higher than 3.5ppm, the HACH Test Kit will not be able to identify it. The use of the comparator disk in the HACH Test Kit was produced by the manufacturer in 2004. Through the years, the colour band located on the disk may have worn off due to overuse or UV damage. The result of the damaged disk may have been the reason why the $\mathrm{HACH}$ test kit did not read the correct Chlorine concentration readings. It is recommended that for each HACH Test Kits, the comparator disk should be stored safely from UV radiation. In addition, the comparator disk should be renewed every year so that they provide accurate readings for those using the test kit.

This study was performed within the lab room (SW1 1230) of BCIT. The lab room contains sufficient lighting when using the HACH and Taylor Test Kit. Unfortunately, during pool inspections, the environment in which the operator or health inspector is using these test kits may not have sufficient lighting similar to the ones at BCIT. The readings of this study may not reflect on what will actually happen during an onsite inspection of the pool 
Free available chlorine constantly reacts with microbes and contaminants that are within the pool water. This would mean that FAC is continuously being used up every moment when it comes into contact with water. The longer each session of the study takes, the more FAC is being used up. As a result, we are unable to produce chlorine levels that are the same constantly in this study. As such, we are unable to compare whether there is a difference between the test kits as Johal (2004) has done in his previous research.

\section{Conclusion}

Through this study, we are able to identify that the three pool test kits (Taylor, $\mathrm{HACH}$, and ColorQ) used in the market have their own limitations and may provide inaccurate readings with regards to pool chemistry. Although the readings of the tests kits are not accurate, the results still provide a general range of how well the pools are running for operators and health inspectors. However, because of how inaccurate the results of FAC and TC are, it would be harder to calculate combined chlorine levels and provide the needed corrective action to ensure that the pool is physically clean and biologically safe.

\section{Recommendations}

For pool operators and health inspectors, it is recommended that test kits should be up to date and the instruments are manufactured recently. Comparator tubes and blocks may have colour deterioration due to overuse and UV radiation. In addition, for the ColorQ test kit, the photometer should be checked regularly for accuracy. If inaccurate results arise from the photometer, it is recommended that they should take it back to the manufacture.

Operators and Inspectors should also use other different indicating factors to identify high levels of combined chlorines within the pool water. Although it is not as accurate as calculating the combined chlorine levels with FAC and TC, it is still a good indication that combined chlorine levels may be high for the pool. These include smell and turbidity of the pool water.

\section{Future Research Suggestions}

Improvements should be made to further improve on this study. It is recommended that actual pool water should be used to ensure that all parameters can be tested upon. In addition, when measuring FAC with actual pool water, it is recommended that assistance would be needed. This is due to the fact that FAC will deplete over time. In order to get accurate results, it is recommended that FAC should be measured within 30 minutes of obtaining the water from the pool.

Other parameters of pool chemistry should also be included in future studies. These include alkalinity and calcium hardness. In addition, bromine accuracy can also be tested to ensure that all types of pools have accurate pool test kits.

\section{Acknowledgements}

The author would like to thank Fred Shaw for arranging the use of supplies to carry out this project; Wini Lao for suggesting this research topic; Bobby Sidhu for providing guidance and advice for this project.

\section{Competing Interests}

The authors declare that they have no competing interests

\section{References}

BC Public Health Act. (2014). SBC 2008, c.28. Retrieved from

http://www.bclaws.ca/Recon/document/ ID/freeside/00 $08028 \quad 01$

Centers for Disease Control and Prevention. (2001). Shigellosis Outbreak Associated With an Unchlorinated Filland-Drain Wading Pool --- Iowa, 2001. MMWR Weekly, 50(37), 797-800.

Centers for Disease Control and Prevention. (2013a). Health Benefits of Waterbased Exercise. Retrieved from http://www.cdc.gov/healthywater/swim ming/health_benefits_water_exercise.ht $\underline{\mathrm{ml}}$

Centers for Disease Control and Prevention. (2013b). Recreational Water Illnesses (RWIs). Retrieved from http://www.cdc.gov/healthywater/swim ming/rwi/

HACH Company. (1991). Pool Master MG/L Test Kit Manual. Retrieved from http://www.hach.com/pool-master-testkit/product-downloads?id $=7640217320$ 
HACH Company. (2013). Pocket Colorimeter ${ }^{\mathrm{TM}}$ Analysis Systems Instruction Manual. Retrieved from www.hach.com/assetget.download.jsa?id=7639982420

Heacock, H., Sidhu, B. (2014a). ENVH 8400 Research Methods. Burnaby, B.C.: British Columbia Institute of Technology, 22 Sept. 2014. Lecture Notes

Heacock, H., Sidhu, B. (2014b). ENVH 8400 Research Methods. Burnaby, B.C.: British Columbia Institute of Technology, 29 Sept. 2014. Lecture Notes

Johal, J. (2004). Swimming Pool Test Kits- Is there really a difference? BCIT student research report for ENVH 8400

Koski, T.A., Stuart, L.S., \& Ortenzio, L.F. (1966). Comparison of Chlorine, Bromine, and Iodine as Disinfectants for Swimming Pool Water. Applied Microbiology, 14(2), 276-279

LaMotte Company. (2014a). ColorQ Photometer for Pool and Spa. Retrieved from http://www.lamotte.com/en/poolspa/digital-testing/colorq

LaMotte Company. (2014b). ColorQ PRO 7 Instructions. Retrieved from http://www.lamotte.com/en/poolspa/digital-testing/colorq/2056.html

LaMotte Company. (2014c). LaMotte Reagent List. Retrieved from http://www.lamotte.com/en/support/reso urces/reagent-list

Pool Regulation. (2012). B.C. Reg. 296/2010. BC Public Health Act. Retrieved from http://www.bclaws.ca/civix/document/id /complete/statreg/296_2010

Pool \& Spa Operator ${ }^{\mathrm{TM}}$ Handbook. (2014a) Pool Water Contamination. Colorado Springs, CO: National Swimming Pool Foundation ${ }^{\circledR}$
Pool \& Spa Operator ${ }^{\mathrm{TM}}$ Handbook. (2014b) Disinfection. Colorado Springs, CO: National Swimming Pool Foundation ${ }^{\circledR}$

Pool \& Spa Operator ${ }^{\mathrm{TM}}$ Handbook. (2014c) Pool \& Spa Water Problems. Colorado Springs, CO: National Swimming Pool Foundation ${ }^{\circledR}$

Sidhu, B. (2014a). ENVH 1143 Pools and Recreational Water. Burnaby, B.C.: British Columbia Institute of Technology, 26 Sept. 2014. Lecture Notes

Sidhu, B. (2014b). ENVH 1143 Pools and Recreational Water. Burnaby, B.C.: British Columbia Institute of Technology, 24 Oct. 2014. Lecture Notes

Taylor Company. (2014a). Reagents.

Retrieved from https://www.taylortechnologies.com/pro ducts_reagents_search.asp

Taylor Company. (2014b). Instruction Number 5121. Retrieved from http://www.taylortechnologies.com/prod ucts_instructions.asp?Type $=$ Number \&N umber $=5121$

Taylor Company. (2014c). Reagent Shelf Life. Retrieved from https://www.taylortechnologies.com/Ch emistryTopicsCM.ASP?ContentID=26

Verma, A., Bolton, F.J., Fiefield, D., Lamb, P., Woloschin, E., Smith, N., \& McCann, R. (2007). An outbreak of E. coli O157 associated with a swimming pool: an unusual vehicle of transmission. Epidemiology and Infection, 135(6), 989-992

World Health Organization. (2006). Guidelines for safe recreational water environments. Geneva: World Health Organization. Retrieved from http://apps.who.int/iris/bitstream/10665/ 43336/1/9241546808 eng.pdf?ua=1 